\section{Data analysis programs for the UNIX operating system}

\section{GARY PERLMAN}

University of California at San Diego, La Jolla, California 92093

The UNIX ${ }^{1}$ operating system is a program development and text editing facility developed at Bell Laboratories (Richie \& Thompson, 1974, 1978). UNIX is a highly interactive operating system, and because of this, it is an ideal environment for data analysis. Although the programs to be described were developed for use on UNIX, they are adaptable for use in other programming environments. In this paper, I will briefly describe how UNIX users control the flow of data through programs, then describe a facility for transforming and formatting data, and, finally, describe three useful programs. These programs are easy to use and require little knowledge of statistics or computers.

\section{USING UNIX}

UNIX users generally sit in front of a terminal at which they repeatedly specify a program, the input to that program, and where the output from the program should be directed. The default input to a program is called the "standard input," and the default output is called the "standard output." If the user does not override where the standard input comes from or where the standard output goes, UNIX assumes the terminal keyboard and screen, respectively, are desired. To input some data to a program that reads from the standard input, a user can redirect the data from a file with the "<" symbol. Thus,

$$
\text { program }<\text { file }
$$

indicates to UNIX that the input to the program "program" is to be read from the file "file" rather than from the terminal keyboard. Analogously, the output from a program can be redirected to a file with the " $>$ " symbol. Thus,

$$
\text { program }<\text { input }>\text { output }
$$

indicates that the program "program" should read its

Much of the inspiration for these programs came from Jay McClelland. The facilities for checking for erroneous inputs were added on the advice of Greg Davidson. These programs were developed while I was supported by a Natural Science and Engineering Research Council of Canada Postgraduate Scholarship. This work was also supported in part by the Office of Naval Research (ONR) under Contract N00014-79-C-0323, NR $157-437$ and by the Advanced Research Projects Agency, monitored by ONR under Contract N00014-79-0515, NR 157-434. input from the file "input" and also that it should write its output to the file "output."

The output from one program can be made the input to another (this action is called pipelining, or piping) using the "p" symbol. Thus,

$$
\text { program } 1<\text { input } \mid \text { program2 }
$$

tells UNIX to run the program "program1" on the file "input" and feed the output from "programl" to another program, "program2." In this case, the final output would be printed on the terminal screen because the final output from "program2" was not redirected. Having the facilities to pipe the output of one program to another allows a user to apply transformations to a master data file until data are in a correct format for input to one of the analysis programs described in a later section.

\section{THE MASTER DATA FILE}

The master data file is a file of lines, each with the same number of alphanumeric fields. These lines are in free format, with fields separated by tabs or spaces for readability. Usually, a master file contains all the data from an experiment. In many cases, a user would not want all the data from this file to be input to a program or might want to transform data prior to input, or both. A program for extracting fields of a line (possibly based on conditions) and for transforming fields is described below.

\section{PROGRAM DESCRIPTIONS}

The following programs were designed with the philosophy that data, in a natural format, can convey all or most of the information a program needs for analysis. With data formatting facilities, the need for specifying the format of the input to most programs all but disappears. Users can implicitly specify design information by putting their data into specific formats.

\section{Formatting and Transforming Data}

DM is a data manipulating program that allows its user to extract columns from a file, possibly based on conditions, and to produce algebraic combinations of columns. To use DM, a user writes a series of expressions, and, for each line of the input, DM reevaluates and prints the values of those expressions. DM provides its user with much of the power of an interpreted generalpurpose programming language, but it is much easier to use. Thus, DM does most any transformation on data one would usually need to write and compile a one-shot program for.

DM allows its user to access the fields on each line 
of its input. Numerical values of fields on a line can be accessed by the letter " $x$ " followed by a column number. Character strings can be accessed by the letter "s" followed by a column number. For example, for the input line:

\section{2 red}

$\mathrm{s} 1$ is the string ' 12 ', $\mathrm{x} 2$ is the number 45.2 (which is not the same as $s 2$, the string ' $45.2^{\prime}$ ), and $s 3$ is the string 'red'.

Column Extraction. Simple column extraction can be accomplished by typing the strings from the columns desired. For example, to extract the third, eighth, first, and second columns (in that order) from "file," one would type:

\section{DM s3 s8 s1 s2 < file}

Algebraic Transformations. Algebraic operations involving the numerical values of fields can be accomplished in a straightforward manner. To print, in order, the sum of the first two columns, the difference of the next two columns, and the square root of the sum of squares of the first and third columns, one would type the expressions:

$$
\begin{gathered}
x 1+x 2 \\
x 3-x 4 \\
(x 1 * x 1+x 3 * x 3) * .5
\end{gathered}
$$

Testing Conditions. Expressions can be conditionally evaluated by testing the values of other expressions. For example, to print the ratio of $\mathrm{xl}$ and $\mathrm{x} 2$, one might want to check the value of $\times 2$ before division and print "error" if $x 2$ is zero. This could be done by the expression:

$$
\text { if } x 2=0 \text { then 'error' else } x 1 / x 2
$$

Or one might want to extract only those lines in which two columns have the same lexical value, as in the case of searching for correct responses. Assuming that the obtained response is in Column 5 and the correct response is in Column 2, this could be accomplished with:

$$
\text { if } s 5=s 2 \text { then INPUT else KILL }
$$

INPUT is a string variable that is equal to the current input line, and KILL is a control primitive that terminates execution for the current line.

Other Features. DM offers a full set of comparison, algebraic, and logical operators. It also features a set of special variables that hold useful information for the user and allow taking control in exceptional conditions. These include: INPUT, the current input line; $N$, the number of fields in INPUT; SUM, the sum of the columns in INPUT; RAND, a uniform random number; NIL, an expression that causes no output; KILL, which terminates evaluation on INPUT and goes to the next line; and EXIT, which terminates all processing.

Limitations. DM allows at most 100 columns in its input or output, although both of these can be changed with the adjustment of a single parameter. DM is the only one of the programs mentioned here that is highly dependent on the UNIX operating system, because it uses a compiler-compiler developed for UNIX users (Johnson \& Lesk, 1978).

\section{ANALYSIS PROGRAMS}

The following programs do not require the usual task of explicitly specifying the characteristics of their input with control cards. Input is assumed to be in a specific yet natural form because of the formatting facilities described above. The idea is to format or transform data from a master data file with DM and pipe the output from DM to one of the following analysis programs. All of the following programs were designed with the user in mind; they allow free format input and provide diagnostic error messages and help options.

\section{Multivariate Analysis of Variance}

ANOVA is a program that does multivariate analysis of variance on designs with within-subjects factors, between-subjects factors, or both. ANOVA allows variable numbers of replications (averaged before analysis) on any factor. Unequal group sizes on betweensubjects factors are also allowed and are solved with the weighted-means solution. The program is based on a method of analysis discussed by Keppel (1973). ANOVA uses an input format unlike conventional programs. The input format is designed so that if a user specifies the role individual data play in the overall design, ANOVA will figure out program parameters that usually need to be explicitly specified, usually with a special control language. This way, the error-prone process of specifying complicated nesting and crossing of factors is taken out of the hands of the user.

Input Format. The input to ANOVA consists of each datum on a separate line, preceded by a list of indexes, one for each factor, that specify the level of each factor at which that datum was obtained. By convention, data are always in the last column, and indexes for the one allowable random factor must appear in the first. Data can be real numbers or integers. Indexes can be any character string of up to seven characters, allowing mnemonic labels to simplify reading cell means. For example:

\section{s5 3 hard 10}

indicates that subject "s5" at level " 3 " of the factor indexed by Column 2 and at level "hard" of the factor 
indexed by Column 3 scored 10. The input to ANOVA consists of a series of lines like the one above. The order of these lines does not matter, so additional data can simply be appended to the end of a file. Replications are coded by having more than one line with the same list of leading indexes. From a series of data lines, ANOVA determines the number of factors, the number of levels of each factor, and whether a factor is within or between subjects, information users are usually required to supply.

Optionally, names of independent and dependent variables can be specified to allow mnemonic labels for the output. For example, in a three-factor design, the call to ANOVA:

\section{ANOVA subjects group difficulty errors}

gives the name "subjects" to the random factor, "group" and "difficulty" to the next two factors, and "errors" to the dependent variable. If names are not specified, default names are used.

Output. The output from ANOVA consists of cell means and standard deviations for each source not involving the random factor, a summary of design information, and an $\mathrm{F}$ table. Sums of squares, degrees of freedom, mean squares, $F$ ratio, and significance level are all reported for each $F$ test.

Limitations and Error Checking. The maximum number of factors ANOVA allows is 10 and the maximum number of levels of any factor is 100 , although both these limitations can be changed by resetting just one parameter. ANOVA checks its input to make sure that data are numerical and that each datum is preceded by a consistent number of indexes. ANOVA will not print its $F$ table if it does not understand the input specification. This can happen if there are missing data or if a subject in a between-subjects factor appears at more than one level of that factor.

An Example. Imagine an experiment in which the two factors of interest are difficulty of material to be learned and amount of knowledge a subject brings with him or her into the experiment. Each subject is given two learning tasks, one easy and one hard, so task difficulty is a within-subjects factor. Assume three subjects are experts in the task chosen and four are novices, so knowledge is a between-subjects factor with unequal group sizes. The dependent variable is the amount of time it takes a subject to correctly work through a problem. A set of fictitious data is shown in Table 1 . The call to ANOVA to analyze these data would probably look like this:

\section{ANOVA subjects difficulty knowledge time $<$ data}

"Data" is the name of the file containing the data in Table 1. The random factor is "subjects," so indexes for it appear in the first column. The dependent measure, here called "time," must appear in the last column. In the second column, "difficulty" is identified as a withinsubjects factor because each subject appears at every level of that factor. In the third column are indexes for level of "knowledge," a between-subjects factor, so identified because no subject appears at more than one level of that factor. The output from the analysis is shown in Table 2.

\section{Multivariate Linear Regression}

REGRESS does multivariate linear regression and correlation. The program assumes its input is a series of lines, each with an equal number of numerical fields, one for each variable. The methods of analysis are based on

Table 1

Fictitious Data

\begin{tabular}{lllr}
\hline S1 & easy & novice & 12 \\
S1 & hard & novice & 22 \\
S2 & easy & novice & 15 \\
S2 & hard & novice & 16 \\
S3 & easy & novice & 10 \\
S3 & hard & novice & 15 \\
S4 & easy & novice & 25 \\
S4 & hard & novice & 30 \\
S5 & easy & expert & 7 \\
S5 & hard & expert & 10 \\
S6 & easy & expert & 12 \\
S6 & hard & expert & 18 \\
S7 & easy & expert & 15 \\
S7 & hard & expert & 14 \\
\hline
\end{tabular}

Table 2

Sample Output from ANOVA, Based on Data in Table 1

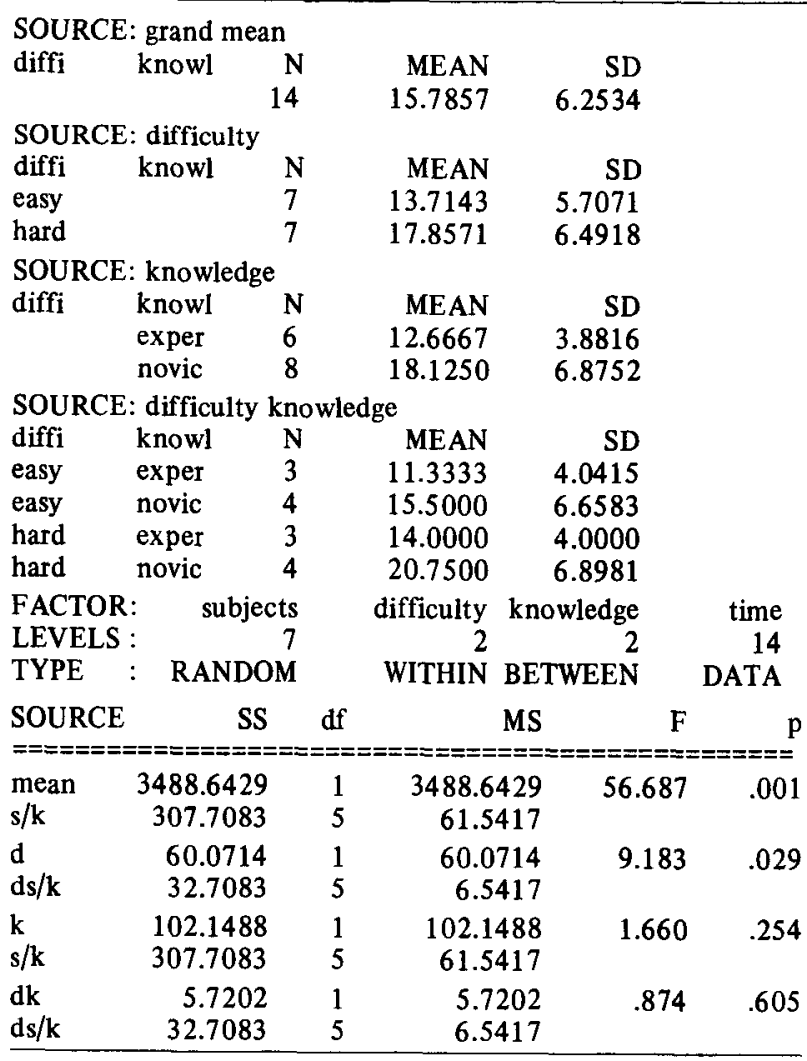


Table 3

Sample Output from REGRESS Based on Data from Table 1

\begin{tabular}{|c|c|c|}
\hline \multicolumn{3}{|c|}{ Analy sis for 7 points of 2 variables: } \\
\hline VARIABLE : & $: \quad$ easy & hard \\
\hline MEAN & 13.714 & 17.857 \\
\hline SD & 5.707 & 6.492 \\
\hline \multicolumn{3}{|c|}{ CORRELATION MATRIX: } \\
\hline easy : & $: \quad 1.000$ & \\
\hline hard & .835 & 1.000 \\
\hline \multicolumn{3}{|c|}{ REGRESSION EQUATIONS: } \\
\hline SLOPES & : & \\
\hline easy & : & .950 \\
\hline hard & .734 & \\
\hline INTERCEPT : & .599 & 4.825 \\
\hline R-squares & .698 & .698 \\
\hline$F(1,5)$ & 11.554 & 11.554 \\
\hline $\mathrm{p}$ & .019 & .019 \\
\hline
\end{tabular}

Note-Read regression equation predicting a variable in the column under that variable's name. Hard $=.950$ easy +4.825 ; easy $=.734$ hard +.599 .

those described by Kerlinger and Pedhazur (1973). Like ANOVA, mnemonic names for each column can be specified.

Output. The output includes means, standard deviations, and cross-correlations for each variable. Optionally, REGRESS does a regression analysis for which it prints (1) a set of regression equations (one for each variable) in which each variable is predicted by all the others, (2) the squared multiple-correlation coefficient for each of these equations, and (3) a significance test (F ratio and significance level) for each multiple $R$.

Limitations and Error Checking. The maximum number of variables is 10 ; however, this can be increased by changing one parameter. REGRESS checks its input to make sure that each line has an equal number of columns and that all fields on a line are numerical. REGRESS will refuse to print regression equations if two variables are perfectly correlated.

An Example. The data in Table 1 include a withinsubjects factor called "difficulty." To see how highly the two levels of this factor are correlated, all those points with "easy" in Column 2 from Table 1 are placed into one column, and all those indexed with "hard" go into a second. The output from REGRESS applied to this input is shown in Table 3 .

\section{Describing a Single Distribution}

DESC is a multipurpose program for almost any analysis on a single distribution of data including summary statistics, frequency counts, and histograms. The formulas for summary statistics are from the SPSS (Nie, Hull, Jenkins, Steinbrenner, \& Bent, 1975). DESC takes option arguments in its call from UNIX to tell it the analyses desired. For example, to print summary statistics and a histogram with a minimum allowable value of zero and an interval width of five, one could type:

\section{DESC S h $\mathrm{m} 0$ i5 < file}

The " $S$ " requests statistics, the " $h$ " requests a histogram, "m0" sets the minimum value to zero, and "is" sets the interval width of the histogram to five. Any of the options in Table 4 can be appended to a call to DESC.

Limitations. DESC sometimes has to store its data, such as when it computes order statistics. When it has to store its data, DESC is limited to 5,000 points; otherwise, no such limit exists. For histograms or frequency tables, the number of bins in the table is limited to 500 . Both these limitations are easily modified.

An Example. Once again referring to the data in Table 1, DESC run on all the data in the last column with the options in the above UNIX command line produces the output in Table 5 .

\section{COMPUTERS AND LANGUAGE}

These programs are written in C (Version 7) (Kernighan \& Richie, 1978), the systems programming language of UNIX (Richie \& Thompson, 1974, 1978). C is a structured programming language similar to ALGOL and Pascal. The programs are all written in a uniform, well commented, highly modularized style, and they share many subroutines, so modification or translation

Table 4

Options Available with DESC

\begin{tabular}{|c|c|}
\hline ptions to format fre & bles: \\
\hline $\mathrm{i}<$ num $>$ & sets the INTERVAL WIDTH to <num $>$ \\
\hline $\mathrm{m}<$ num $>$ & sets the MINIMUM value of the first bin to $<$ num $>$ \\
\hline$M<$ num $>$ & sets the MAXIMUM value to <num $>$ \\
\hline Options used to speci & of table: \\
\hline c & converts $f$ and $p$ options to CUMULATIVE \\
\hline f & table of raw FREQUENCIES \\
\hline $\mathrm{h}$ & HISTOGRAM \\
\hline p & table of PROPORTIONS \\
\hline Options used to sumn & 11a: \\
\hline$m<$ num $>$ & all numbers less than <num> are ignored ON INPLT \\
\hline$M<$ num $>$ & all numbers more then <num > are ignored ON INPUT \\
\hline $\mathrm{s}$ & summary STATISTICS (without order statistics) \\
\hline $\mathrm{S}$ & same as s, but STORE for order statistics \\
\hline $\mathrm{t}<\mathrm{num}>$ & T-TEST of Ho: mean $=\langle$ num $\rangle$ \\
\hline
\end{tabular}


Table 5

Output from "DESC S h m0 is" Run on Data in Table 1

\begin{tabular}{lrrllr}
\hline $\mathrm{n}$ & $=$ & 14 & & & \\
$\min$ & $=$ & 7.000 & $\max$ & $=$ & 30.000 \\
$\#<\min$ & $=$ & 0 & \#>MAX & $=$ & 0 \\
$\mathrm{zmin}$ & $=$ & -1.405 & $\mathrm{zmax}$ & $=$ & 2.273 \\
midpt $=$ & 18.500 & range & $=$ & 23.000 \\
$\mathrm{Q} 1 \mathrm{Q3}$ & $=$ & 18.000 \\
median $=$ & 12.000 & $\mathrm{Q} 3$ & $=$ & 3.000 \\
mean & 15.000 & $\mathrm{qd}$ & $=$ & 6.253 \\
gmean $=$ & 15.786 & sd & $=$ & 13.755 \\
skew $=$ & 14.730 & hmean & $=$ & 2.717 \\
MIDPOINT & FREQ & kurt & $=$ & \\
OUTLIERS & 0 & & & \\
2.500 & 0 & & & \\
7.500 & $3 * * *$ & & & \\
12.500 & $6 * * * * *$ & & & \\
17.500 & $2 * *$ & & & \\
22.500 & $2 * *$ & & & \\
27.500 & $1 *$ & & & \\
\hline
\end{tabular}

of these programs from $\mathrm{C}$ to similar languages is relatively easy. The programs are quite small and can run on most microcomputers. These programs are now running on three DEC computers: the PDP-11/45, the PDP-11/34, and the VAX 11/780; On the PDP-11/45, users also have access to programs from BMD (Dixon, 1975), notably P2V (multivariate analysis of covariance with repeated measures), and $\mathrm{P} 2 \mathrm{R}$ (multiple stepwise linear regression); however, the programs described here are unanimously preferred because of ease of use (free formats, error diagnostics), better response time (roughly a factor of 10 ), and the availability of supporting documentation. One of the reasons for the dramatic differences in response time over the BMD programs is that, on the average, the programs described here are 15 times smaller than their BMD counterparts, even though they are nearly as powerful.

\section{AVAILABILITY}

All of the programs described here, along with supporting documentation and sample inputs and outputs, are available from the author at the Department of Psychology, C009, University of California at San Diego, La Jolla, California 92093. UNIX users can send a magnetic tape or 8-in. floppy disk, along with a prepaid return mailer, to obtain soft-copy versions.

\section{REFERENCES}

Dixon, W. J. BMD-P biomedical computer programs. Berkeley: University of California Press, 1975.

Johnson, S. C., \& Lesk, M. E. Language development tools. Bell System Technical Journal, 1978, 57, 2155-2175.

KePPEL, G. Design and analysis: A researcher's handbook. Englewood Cliffs, N.J: Prentice-Hall, 1973.

Kerlinger, F. N., \& Pedhazur, E. J. Multiple regression in behavioral research. New York: Holt, Rinehart, \& Winston, 1973.

Kennighan, B. W., \& Richie, D. M. The C programming language. Englewood Cliffs, N.J: Prentice-Hall, 1978.

Nie, H. H., Hull, C. H., Jenkins, J. G., Steinbrenner, K. \& BENT, D. H. Statistical package for the social sciences. New York: McGraw-Hill, 1975.

Richie, D. M. \& Thompson, K. The UNIX time-sharing system. Communications of the Association for Computing Machinery, 1974, 17, 365-375.

Richie, D. M., \& Thom pson, K. The UNIX time-sharing system. Bell System Technical Journal, 1978, 57, 1905-1929.

\section{NOTE}

1. UNIX is a trademark of Bell Laboratories.

(Accepted for publication June 26, 1980.) 